\title{
MEASUREMENT OF THE POLARISATION, SPECTRA AND ACCURATE LOCATIONS OF COSMIC X-RAY SOURCES
}

\author{
K. A. POUNDS \\ Dept. Physics, University of Leicester, Leicester, U.K.
}

Following the successful flights of large area survey experiments on three unstabilised Skylark rockets in 1967, 8 and 9, the Leicester Group has now changed the emphasis of its Cosmic X-ray Astronomy programme to the detailed observation of specific sources using attitude-controlled vehicles. Two new Skylark experiments for flights in the current year are described and some details are also given of an X-ray polarimeter/spectrometer being prepared for launch in 1973 on the UK-5 satellite.

Low resolution spectra of the Sco X-1 source have generally been interpreted in terms of optically thin thermal bremsstrahlung with a characteristic temperature of about $4 \mathrm{keV}$. In order to make a definitive check of this hypothesis a large area (220 $\mathrm{cm}^{2}$ ) lithium fluoride Bragg crystal spectrometer has been built, and recently flown, to search for the anticipated line emission of highly ionized iron. To provide maximum sensitivity the crystal scan was severely restricted to two degrees about the Bragg position for the resonance line of helium-like Fexxv at $6.65 \mathrm{keV}$, and a special low background proportional counter detector was fabricated from plastic scintillator, the latter being used for anti-coincidence rejection of cosmic-ray-induced counts. In addition, pulse rise-time discrimination was used, yielding an overall particle background rejection of $96 \%$. This experiment was flown successfully on Skylark 901 from Woomera, South Australia, on 18th March this year. Three spectral scans were obtained in a total exposure of $240 \mathrm{~s}$, but a provisional analysis has revealed no evident emission line. In contrast, an optically thin thermal source with a coronal abundance of iron $\left(3 \times 10^{-5} N_{H}\right)$ would have yielded a line signal $15 \sigma$ above the flight background. The possibility of this result being due to considerable line broadening, arising from multiple electron scattering of the X-ray photons in an extremely dense source $\left(\mathrm{Ne} \sim 10^{16} \mathrm{~cm}^{-3}\right)$, is discussed. A unique feature of this flight was the use of a pair of proportional counters to provide an error signal from which the rocket roll datum was locked onto the Sco X-1 source, the main rocket axis being pointed at the Sun. Some $2 \times 10^{5}$ Sco X-1 counts were recorded from these detectors and have been used to provide a broad-band spectrum from $4-14 \mathrm{keV}$. The analysis of this data is still incomplete and will be used to provide an independent measurement of the 'source temperature' at the time of flight and the possible strength of a broadened emission line consistent with a thermal source model and also with the apparent absence of a narrow feature on the crystal spectrum.

The second rocket payload, now almost complete, consists of a modulation collimator in which the spacing between the pair of identical wire grids is continually varied throughout an observation. The experiment will be flown on a star-pointing (Stage V) Skylark in a few months time. with the aim of measuring the absolute 
positions of a local group of X-ray sources to better than 1 arc min. A proportional counter with $550 \mathrm{~cm}^{2}$ window is mounted looking forward along the longitudinal rocket axis, through the pair of grids. After the rocket has acquired the chosen $\left(M_{v} \leqslant 3\right)$ visible reference star, the rear grid is moved slowly out towards the fixed front grid. As it moves, the X-ray count rate from individual sources within the $20^{\circ} \times 20^{\circ}$ field of view is modulated at a frequency which depends solely on their position relative to the axis of symmetry of the grid pair. The main advantages of the Variable Spring Modulation Collimator over a conventional, scanning egg-box, collimator, are, first, that every source is in view for the whole flight - with a corresponding increase in sensitivity - whilst, secondly, the grid collimators can be made with a much smaller geometrical collimation factor. In the present case, for Skylark 812 , the grids each have a $200 \mu \mathrm{m}$ pitch, with FWHM collimation of 1.8 arc min. This figure corresponds to a modulation frequency difference of unity and, since for most sources it will be possible to determine the fundamental frequency to a fraction of this, the anticipated accuracy of relative source positions is $20-30 \mathrm{arc} \mathrm{sec}$. The observation of the modulated visible light of the bright reference star, with a photomultiplier mounted behind the grids, will be used to relate the X-ray positions in celestial coordinates. Since one observation gives only the source positions in the direction of the grid wires, the rocket will then be rolled through $90^{\circ}$ and a second grid scan made.

The UK-5 satellite will be spin-stabilised with the spin axis being directed within $1^{\circ}$ of any chosen part of the sky by a ground-controlled gas jet system. A number of experiments are being prepared to make detailed observations of X-ray sources, viewing along the pointing axis. One of these is a Bragg crystal spectrometer, being provided by the Leicester Group to make high resolution spectral measurement and to search for X-ray polarisation of specific sources. Each of a pair of crystals, providionally of lithium hydride and graphite, can be set by command to any Bragg angle between 20 and $70^{\circ}$. As the satellite rotates about the spin axis, the X-rays from a source - typically off-axis by a fraction of a degree - will sweep through a small range of incident angles to the crystal face and in doing so will generally scan through the wavelength position of a pre-selected emission line. The observation of the line will be made from the modulation of the reflected photon flux detected in each of a pair of proportional counters. To examine the incident X-rays from a particular source for polarisation, each crystal will be set to Bragg angle $45^{\circ}$. Then, the component of the radiation polarised in the plane of crystal reflection is totally extinguished, while the perpendicular component is reflected. As the crystal rotates about the satellite spin axis, so the total reflected $\mathrm{X}$-ray intensity will be modulated at twice the rotation frequency, the depth of modulation representing the polarisation in the incident beam.

The sensitivity for both polarisation and spectroscopy is set by the precision with which the reflected X-ray source continuum count can be measured. In a 24 hour observation, giving $15 \times 60 \mathrm{~min}$ of data, a polarisation of $2.6 \%$ on the Crab source would be detectable by summing the $2.6 \mathrm{keV}$ and $4.4 \mathrm{keV}$ measurements. Separate limits at these energies would, of course, vary with their intensity. Thus, Cyg X-1 
would be similar to the Crab whilst for M87, some 20 times fainter, the detectable limit in 1 day would be about $45 \%$.

In order to achieve the above sensitivity it will be necessary to allow for the countrate modulation occurring for any off-axis source having a sloping photon-energy spectrum. Thus, for $1{ }^{\circ}$ off-set on Sco X-1, a $12 \%$ spin modulation will occur. However, this will be at twice the period of the polarisation modulation and can be precisely allowed for in the data analysis, using the known source position and overall spectrum.

In the spectroscopy mode, the stronger emission lines predicted for Sco X-1 can be accurately measured in a few minutes observation. For weak sources a similar sensitivity is obtained in an observing period increasing at a rate inversely proportional to the square of the relative source strength, since the cosmic ray background is now dominant. Thus, in a 24 hour observation, a line emission contribution as predicted for Sco X-1 will be detectable from sources 200 times weaker. 